\title{
Design of a Parallel Multi-Filtered Compressive Sampling Model
}

\author{
Jian Sun ${ }^{1, a}$, Jinhe Zhou ${ }^{1, b}$ \\ ${ }^{1}$ School of Information and Communication Engineering, Beijing Information Science and \\ Technology University, Beijing, 100101, China \\ aemail: sjian90@126.com, bemail: zhoujinhe@bistu.edu.cn
}

Keywords: Compressive Sampling; Analog-to-Information; Parallel; Filter

Abstract. Based on the analysis of the existing Analog-to-Information Converter (AIC) under the framework of Compressed Sensing (CS), a novel model called the Parallel Multi-Filtered AIC (PMF-AIC) is proposed. In this model, the ideal integrators in the traditional AIC model are replaced by analog filters with non overlapping pass-bands, as a result that the independence of sampled measurements can be enhanced. Besides, precise integrate devices are not needed in PMF-AIC, so the complexity of the system is reduced. Experiments results show the feasibility of this model.

\section{Introduction}

Traditional sampling theory is based on the Nyquist theorem, which indicates that the lowest sampling frequency of an analog signal should be at least equal to two times the bandwidth of the signal. But current Analog-to-Digital (ADC) technologies cannot meet the demand of sampling task when the bandwidth of an analog signal is ultra-high. Compressed Sensing (CS) $[1,2]$ provide a novel way to solve this problem, which can achieve sampling a signal at a sub-Nyquist sampling rate and exactly recovery the original signal using an optimization method.

AIC is a practical implementation of CS theory. The first proposed AIC model is the Random Demodulation AIC (RD-AIC) [3], which has only one sampling channel. In the RD-AIC, the random modulated signal is sampled after a low-pass filter and the sampled measurements are dependent to some extent and this is bad for the reconstruction of original signal. In order to decrease the dependence among the sampled measurements and the sampling rate, a parallel architecture called Parallel Segmented Compressed Sensing (PSCS) structure is proposed in [4]. In the PSCS structure, a number of parallel Branches of Mixers and Integrators (BMIs) are designed, other AIC architectures such as Modulated Wideband Converter (MWC) [5] and Nyquist Folding Analog-to-Information (NF-AIC) converter [6] have similar parallel structure with PSCS. The integrators in the BMIs should be reset to zero in the beginning of every integration time interval, this increase the system complexity.

In this paper we analyze the function of the filter in RD-AIC and show that a band-pass filter is also adequate in RD-AIC. With this conclusion, referencing the design thought of PSCS, a parallel multi-filtered compressive sampling architecture is proposed. The PMF-AIC has no need of precise integrate devices and can enhance the independence of measurements.

\section{Random Demodulation Analog-to-Information Converter}

The architecture of RD-AIC is shown in Fig. 1. Original analog sparse signal $x(t)$ is modulated by a pseudo-random PN sequence of \pm 1 values, and the alternative speed between +1 and -1 should be faster than the Nquist frequency. After filtered by a low-pass filter, the modulated signal is sampled by a low rate ADC, finally the original signal is reconstructed using the sampled data. Suppose analog signal $x(t)(\mathrm{t} \in(0, \mathrm{~T}))$ can be represented in a linear superposition by a series of analog signals as follow

$$
x(t)=\sum_{n=1}^{N} \alpha_{n} \psi_{n}(t)
$$


where, the coefficient vector $\alpha=\left[\alpha_{1}, \alpha_{2}, \ldots, \alpha_{N}\right]^{T}$ only has a few nonzero elements and the locations and values of these nonzero elements are unknown.

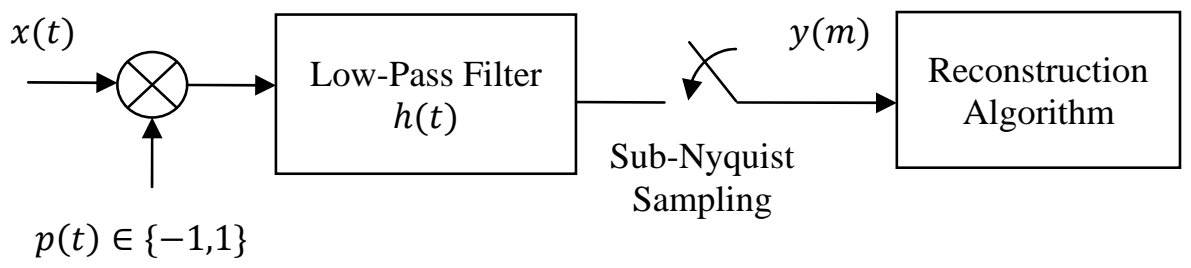

Fig.1. Architecture of RD-AIC

The signal after low-pass filter can be represented as

$y(t)=\int_{-\infty}^{+\infty} x(\tau) p(\tau) h(t-\tau) d \tau$

Suppose the sampling frequency in RD-AIC is $f_{s}$, let $t=\frac{m}{f_{s}}$, where $m \in\{1,2, \ldots, M\}$, then we can get the $m$ th measurement value

$$
y(m)=\sum_{n=1}^{N} \alpha_{n} \int_{-\infty}^{+\infty} \psi_{n}(\tau) p(\tau) h\left(\frac{m}{f_{s}}-\tau\right) d \tau
$$

The above equation can be written as

$y=\Theta \alpha$.

where, $\Theta$ is an $M \times N$ matrix and its $m$ th row and $n$th column element can be written as

$$
\Theta_{m, n}=\int_{-\infty}^{+\infty} \psi_{n}(\tau) p(\tau) h\left(\frac{m}{f_{s}}-\tau\right) d \tau
$$

The matrix $\Theta$ in equation (4) is the sensing matrix in CS theory. Note that $\psi_{n}(t), p_{c}(t)$ and $h(t)$ are known factors. So by following the CS theory [1], we can get $\alpha$ by solving an optimization problem formulated as Eq.(6) and then recovery original signal $x(t)$ using Eq.(1).

$$
\hat{x}=\operatorname{argmin}\|\alpha\|_{0} \quad \text { s.t. } y=\Theta \alpha .
$$

\section{Filter Analysis of RD-AIC}

Random modulation, low-pass filtering and down sampling are three main operations of RD-AIC. Random modulation aims at spreading the features of the signal to the whole spectrum and low-pass filtering aims at selecting the low-frequency part of the modulated signal to extract the feature of original signal.

In some of the AIC architectures such as PSCS and MWC, ideal integrators are implemented in order to smooth the random-modulated signal. But it is impossible to implement an ideal integrator in practice. Paper [7] indicates that a high-fidelity integrator is not required in AIC as long as the impulse response of the analog filter can be characterized very accurately. In fact, the function of an integrator or a low-pass filter is to form a smooth analog signal that can be sampled with a low rate. Such a smooth analog signal contains the feature of the original signal.

As all the frequency components of random-modulated signal may imply features of the original signal, so it is possible to apply a band-pass filter in RD-AIC as long as the filtered signal can be sampled precisely by a low rate ADC. To meet this requirement, the pass band of the band-pass filter should be located on the low frequency part of the spectrum. 


\section{Parallel Multi-Filtered Analog-to-Information Converter}

As band-pass filter is analyzed to be working in the RD-AIC, it is possible to design a parallel structured AIC that applying different filters in different branches. So we proposed the Parallel Multi-Filtered Analog-to-Information Converter (PMF-AIC) which is show in Fig.3.

In each branch of PMF-AIC there is an analog filter after the mixer, each filter is different from the others, in other words, the pass-band of each filter is not overlapped. By setting this, the sampled measurements of each branch are taken from different frequency part of the random modulated signal after mixers. From this perspective, the dependence of different measurements is decreased.

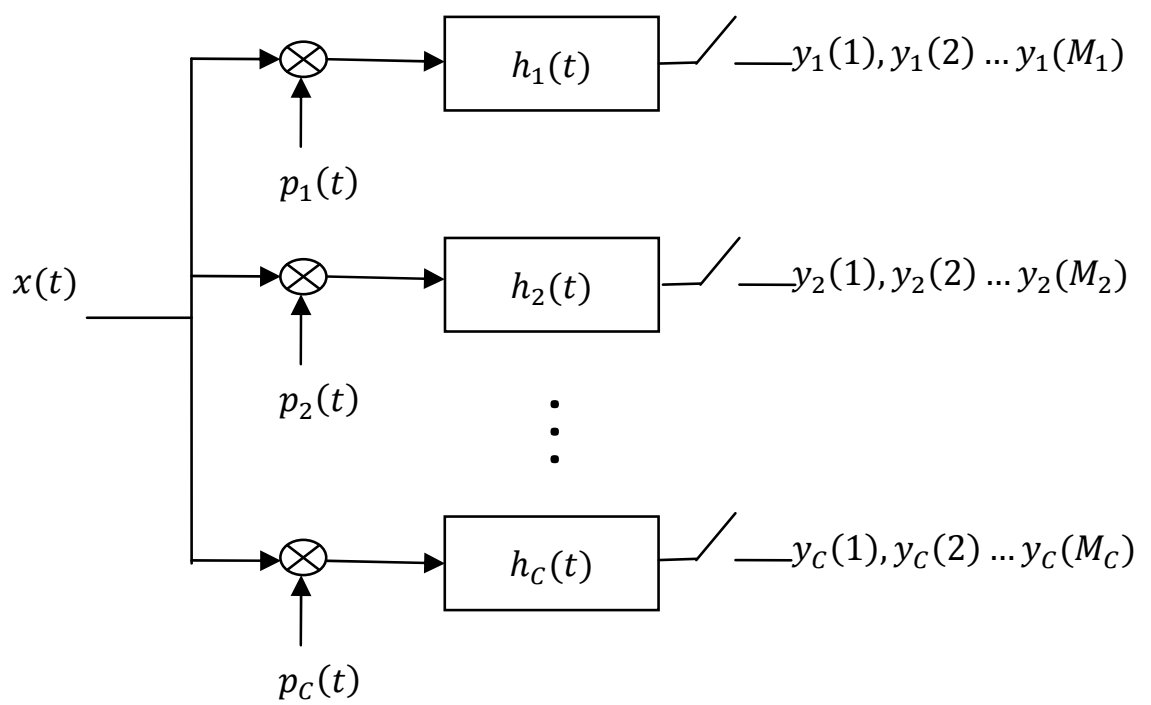

Fig.3. Architecture of PMF-AIC

Assume the total number of branches is $C$ and the measurements number taken in the $c$ th branch is $M_{c}$, then the total number of all the measurements should be

$$
M=M_{1}+M_{2}+\cdots+M_{c} .
$$

Further assume the sampling frequency in the $c$ th branch is $f_{c}$ and the impulse response of the filter in the $c$ th branch is $h_{c}(t)$, then the $m$ th measurement in the $c$ th branch can be expressed as

$$
y_{c}(m)=\sum_{n=1}^{N} \alpha_{n} \int_{-\infty}^{+\infty} \psi_{n}(\tau) p_{c}(\tau) h_{c}\left(\frac{m}{f_{c}}-\tau\right) d \tau .
$$

Formula (8) can be expressed in matrix form as $y_{c}=\Theta_{c} \alpha$, where $y_{c}$ is an $M_{c} \times 1$ vector and $\Theta_{c}$ is an $M_{c} \times N$ matrix with its $m$ th row and $n$th column element is

$$
\Theta_{c, m, n}=\int_{-\infty}^{+\infty} \psi_{n}(\tau) p_{c}(\tau) h_{c}\left(\frac{m}{f_{c}}-\tau\right) d \tau
$$

All the measurements in all the branches can make up a new $M \times 1$ vector

$$
y=\Theta \alpha
$$

where, $y=\left(y_{1}^{T}, y_{2}^{T}, \ldots y_{C}^{T}\right)^{T}, \Theta=\left(\Theta_{1}^{T}, \Theta_{2}^{T}, \ldots \Theta_{C}^{T}\right)^{T}$. Note that equation (10) has the same form with Eq.(4) and then we can solve it with Eq.(6). 


\section{Simulation}

We choose the multi-sinusoid signal as the test original signal and the highest frequency of original signal is $5 \mathrm{GHz}$. Five PMF-AICs with the branch number from 1 to 5 are compared in the simulation. The pass-bands of filters used in each PMF-AIC are shown in Table.1. Note that a PMF-AIC with just one branch shown in Table. 1 is equivalent to a RD-AIC. An orthogonal matching pursuit algorithm (OMP) [8] is used to recovery the original signal in the simulation.

Table.1. Pass-bands of filters used in each PMF-AIC

\begin{tabular}{|c|l|}
\hline Branch number & \multicolumn{1}{|c|}{ Pass-band of filters $(\mathrm{MHz})$} \\
\hline 1 & $(0-200)$ \\
\hline 2 & $(0-200),(400-500)$ \\
\hline 3 & $(0-200),(400-500),(500-600)$ \\
\hline 4 & $(0-200),(400-500),(500-600),(600-700)$ \\
\hline 5 & $(0-200),(400-500),(500-600),(600-700),(700-800)$ \\
\hline
\end{tabular}

The simulation results of five different PMF-AICs are shown in Fig.4. Note that the sampling frequency labeled in x-axis represents the sum of sampling frequency in all the branches of a PMF-AIC. The actual sampling frequency in each branch is smaller than labeled except when the branch number is 1 . As we can see that the performances are lifting when the branch number is larger than one. Unfortunately, the extent of performance lifting is becoming smaller when increase the number of branches, this is may be due to the upper limit of the reconstruction algorithm.

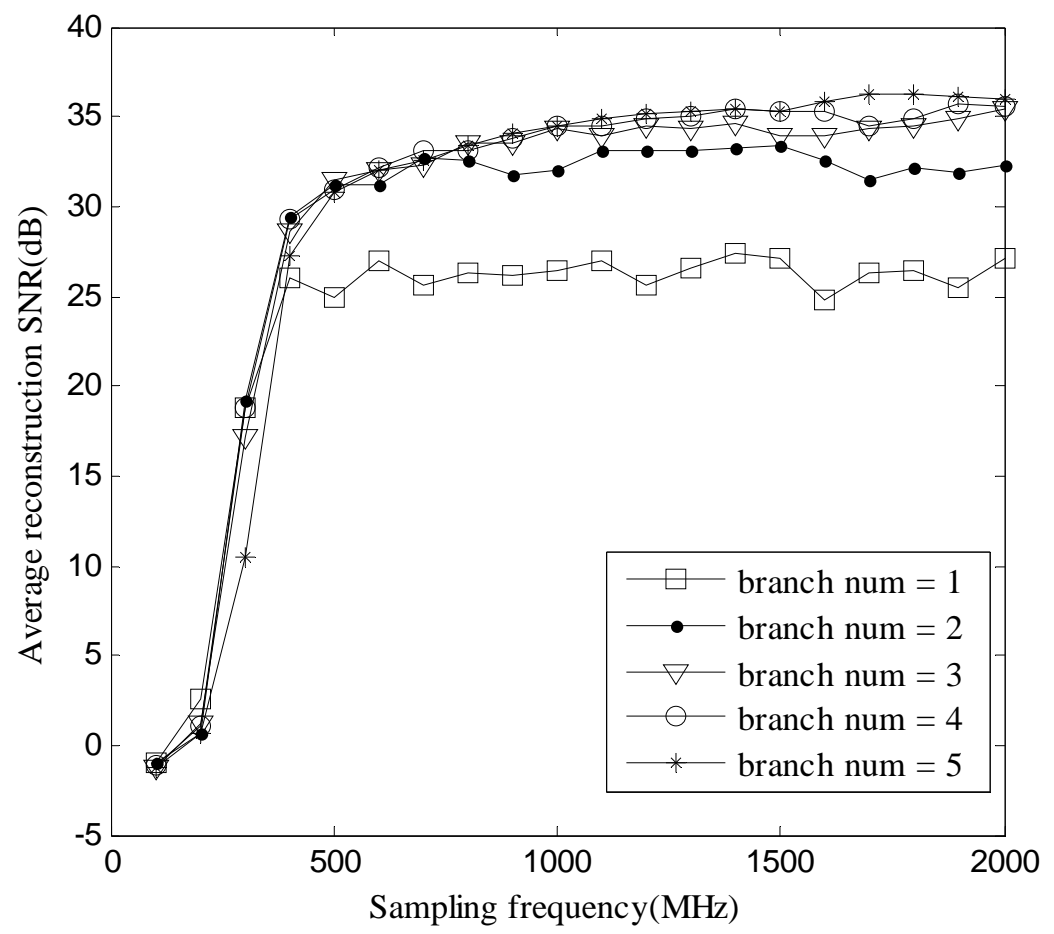

Fig.4. Simulation results

\section{Conclusions}

In this paper we analyze the function of the filter in RD-AIC and show that a band-pass filter is also adequate in RD-AIC. A parallel multi-filtered compressive sampling model is proposed and the proposed PMF-AIC has lower complexity. In the simulation, original signal are successfully reconstructed under the framework of PMF-AIC. Future work may concentrate on exploring more detailed design of this architecture of AIC. 


\section{Acknowledgements}

This work was supported by the Science and Technology Program of Beijing Municipal Education Commission (KZ 201511232036) and the National Natural Science Foundation of China (61271198).

\section{References}

[1] D. L. Donoho: IEEE Transactions on Information Theory, 2006 42(4) 1289-1306.

[2] S. Qaisar, R. Bilal, W. Iqbal, M. Naureen, S. Lee: Journal of Communications and Networks, 2013 15(5) 443-456.

[3] S. Kirolos, J. Laska, M. Wakin, M. Durte, D. Baron, T. Ragheb, Y. Massoud, R. Baraniuk. Analog-to-Information Conversion via Random Demodulation [C], IEEE Dallas/CAS Workshop on Design, Applications, Integration and Software, 2006 71-74.

[4] Y. Zhuizhuan, Hoyos, Sebastian, B.M. Sadler. Mixed-signal Parallel Compressed Sensing and Reception for Cognitive Radio [C]. IEEE International Conference on Acoustics, Speech and Signal Processing, 2008. 3861-3864.

[5] T. Michaeli, Y. Eldar, A. Elron: IEEE Transactions on Signal Processing, 2012 60(3) 1121-1133.

[6] R. Maleh, G. Fudge, F. Boyle, P.E. Pace: IEEE Journal on Emerging and Selected Topics in Circuits and Systems, 2012 2(3) 564-578.

[7] J.A. Tropp, J.N. Laska, M.F. Duarte, J.K. Romberg, R. Baraniuk: IEEE Transactions on Information Theory. 2010 56(1) 520-544.

[8] L. Jia, W. Zhaojun, W. Qiang, L. Yipeng. Greedy Orthogonal Matching Pursuit Algorithm for Sparse Signal Recovery in Compressive Sensing [C], IEEE International Conference on Instrumentation and Measurement Technology. 2014 1355-1358. 